\title{
Structure and Menthone Encapsulation of Corn Starch Modified by Octenyl Succinic Anhydride and Enzymatic Treatment
}

\author{
Xuan Ji, ${ }^{1,2,3}$ Jing Du, ${ }^{1,2,3}$ Jiaying Gu, ${ }^{2}$ Jie Yang, ${ }^{1,2,3}$ Li Cheng, ${ }^{1,2,3}$ Zhaofeng Li, $^{1,2,3}$ \\ Caiming $\mathrm{Li}^{1,2,3}$ and Yan Hong $\mathbb{D}^{1,2,3}$ \\ ${ }^{1}$ Key Laboratory of Synthetic and Biological Colloids, Ministry of Education, Wuxi 214122, Jiangsu Province, China \\ ${ }^{2}$ School of Food Science and Technology, Jiangnan University, Wuxi 214122, Jiangsu Province, China \\ ${ }^{3}$ Collaborative Innovation Center of Food Safety and Quality Control, Jiangnan University, Wuxi 214122, \\ Jiangsu Province, China
}

Correspondence should be addressed to Yan Hong; hongyan@jiangnan.edu.cn

Received 3 February 2022; Accepted 15 February 2022; Published 7 March 2022

Academic Editor: Tao Feng

Copyright $(92022$ Xuan Ji et al. This is an open access article distributed under the Creative Commons Attribution License, which permits unrestricted use, distribution, and reproduction in any medium, provided the original work is properly cited.

\begin{abstract}
In order to improve the ability of starch to absorb menthone, corn starch was modified by enzymatic treatment (amyloglucosidase and $\alpha$-amylase) combined with octenyl succinic anhydride (OSA) esterification. The oil absorption rate of starch modified by enzymatic treatment followed by OSA (P-OSA) reached $101.33 \%$, whereas that of samples with reverse action sequences (OSA-P) was only 59.67\%. The degree of substitution of OSA-P was also generally lower than that of P-OSA. At high OSA addition, OSA-P had a smaller specific surface area with fewer pores because octenyl succinic (OS) groups impeded the enzymatic treatment. Compared with OSA-P, the lamellar structure of P-OSA is sparser and less ordered. Owing to its pores, P-OSA was beneficial for the reaction to occur inside the granules, which was observed by Raman spectroscopy and laser confocal microscopy. At high OSA addition, the loading of P-OSA to menthone could reach $64.34 \mathrm{mg} / \mathrm{g}$.
\end{abstract}

\section{Introduction}

Porous starch is characterized by numerous dents or pores extending to the interior of starch granules. It has higher absorption and slow-release properties than native starch owing to its higher specific surface area. In recent years, porous starch has attracted increasing attention due to its low price, valuable functions, and potential applications [1]. Several approaches, including physical, chemical, enzymatic, and synergic methods, have been used to promote the formation of pores in starch [2]. Porous starch prepared by traditional physical methods, such as mechanical extrusion, microwave, and ultrasonic methods, has a low yield and nonuniform pore size, whereas those derived from chemical methods, including solvent exchange, acid hydrolysis, and molecular insertion, result in porous starch with limited absorption capacity and formation of organic solvent residues during the preparation process. Enzyme catalysis has been widely used for the preparation of porous starch because of the advantages of mild reaction conditions, high catalytic efficiency, and substrate specificity [3].

The absorption capacity of porous starch is influenced by the nature of the raw material, the method of preparation, the absorption conditions, and the nature of the adsorbate, while the absorption capacity per gram of porous starch is generally in the tens of milligrams [4]. Because starch is a hydrophilic substance, porous starch has a weaker absorption capacity for hydrophobic substances than for hydrophilic substances. To improve the porous starch absorption capacity of hydrophobic substances, hydrophobic groups can be introduced by chemical modification. Owing to its hydrophobicity and steric contribution, octenyl succinic anhydride (OSA) can be combined with starch to enhance its hydrophobicity, encapsulation, and emulsification properties [5]. Several studies have explored the combination of porous treatment with esterification modification on starch granules [6-8]. Such studies demonstrated that OSAmodified porous starch has significantly enhanced capacity 
to absorb oils; thus, it could be applied to adsorb hydrophobic substances. Moreover, the presence of pores affects the reaction of the OSA reagent during the esterification modification process, which in turn impacts on the structure and properties of the starch.

Porous starch is mainly used for the absorption of hydrophilic substances, but its ability to adsorb hydrophobic substances is relatively weak. OSA, as a common emulsifier, can impart amphiphilicity to starch and improve its absorption ability to hydrophobic substances. However, there are few studies on the effect of esterification modification on the absorption performance of porous starch and its mechanism, and there is a lack of comparison of the effects of the two reversing treatments on the structure and properties of starch. In this study, amyloglucosidase and $\alpha$-amylase were used to prepare porous starch. The differences in the absorption properties of hydrophobic porous starches prepared by reversing the order of the two treatments were compared, and the influence of starch structure that caused the differences in absorption properties was investigated. Further, hydrophobic porous starch was applied to load menthone.

\section{Materials and Methods}

2.1. Materials. Native corn starch (moisture: $12.36 \mathrm{~g} / 100 \mathrm{~g}$; amylose content: $21.73 \pm 0.81 \%$ ) was provided from Juneng Golden Corn Co., Ltd. (Shandong, China). Amyloglucosidase (EC 3.2.1.3, 20,000 U/mL) was purchased from Genencor International (Palo Alto, CA, USA), and $\alpha$-amylase (EC 3.2.1.1, $10,000 \mathrm{U} / \mathrm{mL}$ ) was purchased from Sukahan (Weifang) BioTechnology Co., Ltd. Octenyl succinic anhydride (OSA) was provided by Huahao Huafeng Co., Ltd. (Guangdong, China). Menthone was purchased from Shanghai Aladdin Biochemical Technology Co., Ltd. Other reagents were purchased from China National Pharmaceutical Group Co., Ltd. (Shanghai, China) and were all of analytical grade.

\subsection{Preparation of Hydrophobic Porous Starch}

2.2.1. Preparation of Porous Starch. Porous starch preparation was based on the method described by Zhang et al. [9] with some modifications. First, a weighed quantity of native corn starch was suspended in phosphate buffer $(\mathrm{pH} 5.5,30 \%$ $\mathrm{w} / \mathrm{w})$. A mixture $(2: 1, \mathrm{v} / \mathrm{v})$ of glucoamylase and $\alpha$-amylase was added to the starch slurry at $2.0 \%(\mathrm{v} / \mathrm{w})$ and was incubated at $50^{\circ} \mathrm{C}$ for $6 \mathrm{~h}$. After the enzymatic reaction, the $\mathrm{pH}$ was adjusted to 3.5 by adding $0.1 \mathrm{M}$ hydrochloric acid to inactivate the enzymes, and the solution was neutralized to pH 7.0 with $0.1 \mathrm{M} \mathrm{NaOH}$ solution. Next, the mixture was filtered and the precipitate was washed three times with deionized water. The final product was dried, milled, and stored for further use.

2.2.2. Preparation of OSA Starch. OSA starch was prepared based on the method described by Song et al. [10] with some modifications. A certain mass of starch was stirred and suspended in deionized water $(30 \%, w / w)$ at $35^{\circ} \mathrm{C}$. The $\mathrm{pH}$ of the suspension was adjusted up to 8.5 by adding $3 \% \mathrm{NaOH}$ solution. A weighed quantity of OSA $(3,6$, and $9 \%$ of the dry starch basis) was diluted with absolute ethanol $(1: 3, \mathrm{v} / \mathrm{v})$ added slowly over a period of $2 \mathrm{~h}$ while maintaining the $\mathrm{pH}$ at 8.5. The reaction was allowed to continue for additional $2 \mathrm{~h}$, after which the $\mathrm{pH}$ was adjusted to 6.5 with $3 \% \mathrm{HCl}$ solution. The mixture was centrifuged and washed twice with deionized water and twice with $70 \%$ aqueous alcohol. The precipitate was oven-dried at $40^{\circ} \mathrm{C}$ for $24 \mathrm{~h}$ and then passed through a 100 -mesh sieve.

OSA-modified porous starch (P-OSA) samples were prepared using corn starch that was first transformed into porous starch and then modified with OSA, whereas OSA-P samples were prepared with reversed treatment sequences (corn starch was first modified with OSA and then hydrolyzed by enzymes).

2.3. Degree of Substitution. The OSA content in starch granules is indicated by the degree of substitution (DS). DS was examined according to a previously described method with some modifications [5]. The starch sample $(5 \mathrm{~g})$ was dispersed in $2.5 \mathrm{M} \mathrm{HCl}$ /isopropyl alcohol solution $(25 \mathrm{~mL})$ by stirring for $30 \mathrm{~min}$. An aqueous $90 \%(\mathrm{v} / \mathrm{v})$ isopropanol solution $(100 \mathrm{~mL})$ was added, and the mixture was stirred for $10 \mathrm{~min}$. After filtration, the residue was washed with $90 \%$ isopropanol until no more $\mathrm{Cl}_{2}$ could be detected (using $0.1 \mathrm{M} \mathrm{AgNO}_{3}$ solution). The starch was redispersed in $300 \mathrm{~mL}$ of deionized water in a boiling water bath for $20 \mathrm{~min}$. The starch was titrated with a $0.1 \mathrm{M} \mathrm{NaOH}$ solution using phenolphthalein as an indicator. Native starch was used as blank control. The DS was calculated according to the following equation:

$$
\mathrm{DS}=\frac{0.162 \times(A \times M) / W}{1-[0.210 \times(A \times M)] / W},
$$

where $A$ is the titration volume of $\mathrm{NaOH}$ solution $(\mathrm{mL}), M$ is the molarity of the $\mathrm{NaOH}$ solution, and $W$ is the dry weight (g) of the OSA starch.

\subsection{Absorption Properties}

2.4.1. Oil Absorption Rate. A quantity of starch was dried at $105^{\circ} \mathrm{C}$ for $4 \mathrm{~h}$ and immersed in $30 \mathrm{~mL}$ of soybean salad oil. After stirring at $25^{\circ} \mathrm{C}$ for $30 \mathrm{~min}$, the mixture was placed in a sand core funnel of known mass and filtered until no oil drops fell. The total mass of the sand core funnel and starch was weighed, and the oil absorption rate was calculated according to the following equation:

$$
\text { oil absorption rate }(\%)=\frac{m_{2}-m_{1}-m_{0}}{m_{1}} \times 100,
$$

where $m_{0}$ and $m_{1}$ represent the weight of the sand core funnel and the starch, respectively, and $m_{2}$ is the weight of the mixture after filtration.

2.4.2. Absorption of Methylene Blue. The absorption of methylene blue (MB) of starch samples was measured in terms of the method described by Xie et al. [11] with some 
modifications. The starch samples $(0.5 \mathrm{~g})$ were dried at $105^{\circ} \mathrm{C}$ for $4 \mathrm{~h}$ and immersed in $30 \mathrm{~mL}$ of $\mathrm{MB}$ solution $(40 \mathrm{mg} / \mathrm{L})$. After stirring for $2 \mathrm{~h}$, the samples were centrifuged at $3,000 \mathrm{rpm}$ for $10 \mathrm{~min}$, and the absorbance of the supernatant was measured at $665 \mathrm{~nm}$ using a spectrophotometer (T-6V; Persee Analytics, Auburn, CA, USA). The equilibrium absorption capacity was calculated according to the following equation:

$$
Q_{e}=\frac{V\left(C_{0}-C_{e}\right)}{m},
$$

where $Q_{e}$ represents the equilibrium absorption capacity of starch sample $(\mathrm{mg} / \mathrm{g}), \mathrm{C}_{0}$ and $C_{e}$ represent the initial and equilibrium concentrations of $\mathrm{MB}$ solution $(\mathrm{mg} / \mathrm{L})$, respectively, and $V$ and $m$ represent the volume of MB solution $(\mathrm{mL})$ and the weight of starch sample $(\mathrm{g})$, respectively.

2.5. Contact Angle. The hydrophobicity of the starch samples is indicated by the magnitude of the contact angle. The starch samples were pressed into sheets. The contact angle was measured using an OCA15EC optical contact angle meter equipped with a CDD camera and WINDROP software, and the measurement method was a sitting drop method with a volume of $2.5 \mu \mathrm{L}$ of injection water [12].

2.6. Starch Granule Morphology Assessment. The morphology of starch samples was observed by using a field-emission scanning electron microscope (SU8100; Hitachi Int., Japan) as described by Xie et al. [2]. Starch samples were spilled on a carrier table with a silver plate and coated with a layer of gold in vacuum prior to observation. Samples were examined at an accelerating voltage of $1.0 \mathrm{kV}$, and images were taken at 2000x and 5000x magnifications.

\subsection{Specific Surface Area and Pore Diameter Assessment.} The specific surface area and pore diameter of the starch samples were estimated using the Autosorb-iQ surface area and pore size analyzer (Quantachrome Instruments, Boynton Beach, FL, USA) by measuring the absorption of liquid nitrogen. Before measurement, the starch samples were dried under vacuum at $150^{\circ} \mathrm{C}$ for $2 \mathrm{~h}$. The isotherm of nitrogen absorption-desorption at liquid nitrogen temperature $(77.35 \mathrm{~K})$ was determined. The specific surface area and the pore size were analyzed by the Barrett-Joyner-Halenda method [13].

2.8. Laminar Structure Evaluation. The laminar structure of the starch samples was determined by small-angle X-ray scattering (SAXSpoint 2.0, Anton Paar, Graz, Austria). The starch samples were configured as starch paste with $50 \%$ moisture content equilibrated at room temperature for $24 \mathrm{~h}$. The acceleration voltage was $50 \mathrm{kV}$, and the current was $1 \mathrm{~mA}$. The scattering intensity $I(q)$ was obtained as a function of the scattering vector $q$ by radially averaging each homogeneous scattering intensity and subtracting the blank group [14].
2.9. Relative Crystallinity. The crystalline features were analyzed using an X-ray diffractometer (Bruker AXS, D2 PHASER, Germany). The diffraction angle (2 $\theta$ ) ranged from 4 to $40^{\circ}$. The scanning speed was set to $2^{\circ} / \mathrm{min}$ with a step size of $0.05^{\circ}$. The relative crystallinity was calculated using JADE 7.0 software (Materials Data Inc., Livermore, USA) based on the ratio of the area of the crystalline peaks to the total area of the diffractogram.

\subsection{Distribution of Octenyl Succinic Groups on Starch Granules}

2.10.1. Raman Spectroscopy Analysis. Spectral data were recorded in the $400-2,000 \mathrm{~cm}^{-1}$ range with a $532 \mathrm{~nm}$ laser using LabRAM HR Evolution (HORIBA Jobin Yvon SAS, Longjumeau, France). An area of $1.2 \mathrm{~mm} \times 1.2 \mathrm{~mm}$ was selected, and 100 points and two spectral images were randomly acquired with a single point acquisition time of $15 \mathrm{~s}$, an accumulation time of $2 \mathrm{~s}$, and a Real Time Display time of $2 \mathrm{~s}$. The synthesized two-dimensional images in the $x$ and $y$ directions provided spatial information, while the third dimension $(z)$ indicated intensity [15].

2.10.2. Laser Confocal Microscopy Analysis. Distribution of the octenyl succinic (OS) groups within the starch granules was determined using a confocal laser scanning microscope (LSM880; Carl Zeiss, Oberkochen, Germany) equipped with an argon ion laser, as previously described $[16,17]$. The lens used was $40 \times / 1.25$, and the gas laser argon laser emission wavelength was $514 \mathrm{~nm}$. The samples $(0.5 \mathrm{~g})$ were suspended in $30 \mathrm{~mL}$ of deionized water. The starch suspension at $\mathrm{pH} 8.0$ was stained with $1 \% \mathrm{MB}$ solution. The mixture was incubated in a shaking water bath at room temperature for $8 \mathrm{~h}$, and the excess dye was removed with methanol.

2.11. Preparation of Menthone Complexes. A certain mass of starch sample was weighed in a centrifuge tube with cap, about 2-3 times the mass of menthone was added, shaken for a period of time to make it dispersed, and then centrifuged at $3000 \mathrm{r} / \mathrm{min}$ for $10 \mathrm{~min}$, and the precipitate was washed quickly with anhydrous ethanol to remove the unadsorbed menthone. The precipitate was dried to obtain the starchmenthone complex.

2.12. Menthone Loading Determination. After weighing $100 \mathrm{mg}$ of the complex and dispersing it in $5 \mathrm{ml}$ of methanol, the mixture was sealed, sonicated for $30 \mathrm{~min}$, left overnight to dissolve menthone in methanol, and then centrifuged at $4000 \mathrm{r} / \mathrm{min}$ for $10 \mathrm{~min}$ to obtain a clarified supernatant. The absorbance of the supernatant was measured, and the content of menthone in the complex was calculated using the regression equation.

2.13. Statistical Analysis. All experiments were performed at least in triplicate. All results were expressed as mean\pm standard deviation (SD). Data were analyzed by one-way analysis of variance (ANOVA) followed by Tukey's test using 
the SPSS 17.0 statistical software program (SPSS Inc., Chicago). Values of $P<0.05$ were considered statistically significant.

\section{Results and Discussion}

3.1. Absorption Properties, Contact Angles, and DS. The starch absorption capacity of hydrophobic substances was determined by the absorption of oil and MB (Table 1). The presence of pores increased the oil absorption rate by 3 -fold that of corn starch; however, no significant increase in oil absorption was observed for OSA starch produced by esterification modification only. When PS was esterified to produce P-OSA, it was observed that P-OSA had a higher oil absorption capacity than PS, and the oil absorption rate gradually increased with the further addition of OSA. Compared with PS, the enhancement of the absorption performance of P-OSA prepared at lower OSA additions (3\% and 6\%) was not significant, which was due to the fact that there were fewer OS groups attached to the starch granules at this time, so the absorption of hydrophobic substances could not be significantly increased. A significant improvement in the absorption of hydrophobic substances was observed when the amount of OSA addition was increased to $9 \%$. In contrast, the oil absorption of OSA$\mathrm{P}$ was lower than that of P-OSA. This was related to the different hydrophobic features of the samples. The interfacial wettability of solid granules can reveal the hydrophobicity of the granules. After hydrolysis of two enzymes and esterification modification, the water-oil interface contact angle of the starch granules increased (Table 1). The contact angle of the products increased with the increase of OSA addition under the same enzymatic conditions, indicating that the presence of long-chain alkenyl groups increased the hydrophobicity of the starch, which would be beneficial to increase the affinity of the starch granules to oil. In contrast, the contact angle of OSA-P was lower than that of P-OSA, and this difference led to the difference in the absorption capacity of the samples on the oil. However, the contact angle was not the only indicator affecting the absorption capacity, which resulted in a lower contact angle for PS samples than OSA samples, but a higher oil absorption capacity for PS samples than OSA samples. The contact angle of OSA9-P sample was the second highest, but its oil absorption capacity was the lowest from all dual modified samples. The causes of this phenomenon were further analyzed and discussed through the observation of starch granule morphology and specific surface area values. The absorption capacity of MB showed a similar variation pattern. The amount of MB adsorbed by porous starch was greatly increased compared with that of corn starch [18]. By comparing the DS values of different samples, the DS values after compound modification were smaller than those of OSA starch at the same OSA addition, regardless of the treatment sequences. This phenomenon may be due to the removal of amorphous regions during pretreatment [19]. Notably, the DS of OSA-P was lower than that of P-OSA with the same amount of OSA added. The decrease of DS indicated the decrease of OSA content in starch granules, which suggests that it was difficult to avoid the part with OS groups being cut off when enzymatically digesting OSA starch.

3.2. Morphology of Starch Granules. Micrographs of the starch samples are shown in Figure 1. Native corn starch exhibited a spherical granular structure with a smooth surface. P-OSA and OSA-P showed a granular morphology with pores distributed on the surface, and different treatment sequences resulted in different pore distribution outcome. The degree of starch morphological variation is highly dependent on the source and properties of the starch, which is in turn due to the differences in the susceptibilities among the starches [18]. Field-emission scanning electron microscopy (FESEM) data showed that the formation of the pores was not effective when esterification modification was performed first. The number of particles with pores in the field of view gradually decreased in the presence of increasing amount of OSA. When OSA (9\%) starch was treated enzymatically, only a small portion of the starch formed a structure with pores, which resulted in a weak absorption capacity of OSA9-P. The increase in the OS groups hindered the formation of additional pores. In contrast, a prior enzymatic treatment followed by OSA modification did not have a significant negative impact on the presence and distribution of pores. This was consistent with Chang et al. [6] report, which suggested that heat during the esterification reaction may alter the structure of pores already formed before the esterification modification.

3.3. Specific Surface Area and Pore Size. Data on the specific surface area and pore size of starch samples with different sequences of OSA modification and enzymatic treatment are shown in Table 2. Compared with porous starch, the specific surface area of P-OSA decreased, whereas the pore size increased after OSA modification. With increased OSA amount, the specific surface area of P-OSA also tended to increase, but the pore size gradually decreased. This may be due to the temperature during the reaction between OSA and the porous starch, as well as the flaking reaction of organic solvents [6]. P-OSA9 had great differences on surface area and pore size from P-OSA3 and P-OSA6. This may be due to the high concentration of OSA during the reaction process which caused the starch granules to swell, bringing about a higher specific surface area and affecting the pore size. For P-OSA, the higher specific surface area had a positive effect on its absorption performance. Although the pore size of the starch decreased at high OSA addition, the higher specific surface area and the presence of hydrophobic groups at this time ensured the superior absorption performance of the samples.

Comparing the specific surface area and pore size of starch samples obtained from two different treatment sequences, it was found that the specific surface area of OSA-P was smaller than that of P-OSA, but the pore sizes were similar. This could be related to the previous FESEM imaging data that the surface pores of OSA-P were less than those of P-OSA, which resulted in a smaller specific surface 
TABLE 1: Absorption properties, DS, and contact angle of CS, PS, P-OSA, and OSA-P.

\begin{tabular}{lcccc}
\hline Samples & Oil $(\%)$ & MB $(\mathrm{mg} / \mathrm{g})$ & DS & Contact angle $\left(^{\circ}\right)$ \\
\hline CS & $25.33 \pm 7.50^{\mathrm{e}}$ & $23.23 \pm 0.31^{\mathrm{j}}$ & - & $19.9 \pm 0.35^{\mathrm{h}}$ \\
PS & $82.67 \pm 4.72^{\mathrm{bc}}$ & $42.53 \pm 0.17^{\mathrm{f}}$ & - & $60.7 \pm 0.37^{\mathrm{g}}$ \\
OSA3 & $29.33 \pm 6.03^{\mathrm{e}}$ & $25.50 \pm 0.10^{\mathrm{i}}$ & $0.0122 \pm 0.0023^{\mathrm{d}}$ & $63.5 \pm 0.39^{\mathrm{g}}$ \\
OSA6 & $34.00 \pm 7.81^{\mathrm{e}}$ & $34.73 \pm 0.56^{\mathrm{h}}$ & $0.0252 \pm 0.0023^{\mathrm{b}}$ & $74.53 \pm 0.41^{\mathrm{f}}$ \\
OSA9 & $39.67 \pm 6.81^{\mathrm{e}}$ & $40.76 \pm 0.66^{\mathrm{g}}$ & $0.0314 \pm 0.0006^{\mathrm{a}}$ & $96.6 \pm 0.72^{\mathrm{e}}$ \\
P-OSA3 & $88.00 \pm 5.29^{\mathrm{abc}}$ & $45.32 \pm 0.23^{\mathrm{e}}$ & $0.0054 \pm 0.0003^{\mathrm{e}}$ & $125.3 \pm 4.6^{\mathrm{c}}$ \\
P-OSA6 & $92.67 \pm 3.51^{\mathrm{ab}}$ & $49.66 \pm 0.09^{\mathrm{d}}$ & $0.0161 \pm 0.0015^{\mathrm{cd}}$ & $127.4 \pm 2.1^{\mathrm{c}}$ \\
P-OSA9 & $101.33 \pm 6.43^{\mathrm{a}}$ & $57.16 \pm 0.11^{\mathrm{a}}$ & $0.0232 \pm 0.0007^{\mathrm{b}}$ & $143.3 \pm 0.3^{\mathrm{a}}$ \\
OSA3-P & $79.33 \pm 2.52^{\mathrm{bc}}$ & $42.04 \pm 0.70^{\mathrm{f}}$ & $0.0051 \pm 0.0008^{\mathrm{e}}$ & $75.6 \pm 3.1^{\mathrm{f}}$ \\
OSA6-P & $74.67 \pm 4.93^{\mathrm{cd}}$ & $51.91 \pm 0.27^{\mathrm{c}}$ & $0.0136 \pm 0.0015^{\mathrm{cd}}$ & $117.0 \pm 0.7^{\mathrm{d}}$ \\
OSA9-P & $59.67 \pm 7.02^{\mathrm{d}}$ & $54.43 \pm 0.36^{\mathrm{b}}$ & $0.0172 \pm 0.0013^{\mathrm{c}}$ & $136.2 \pm 0.2^{\mathrm{b}}$ \\
\hline
\end{tabular}

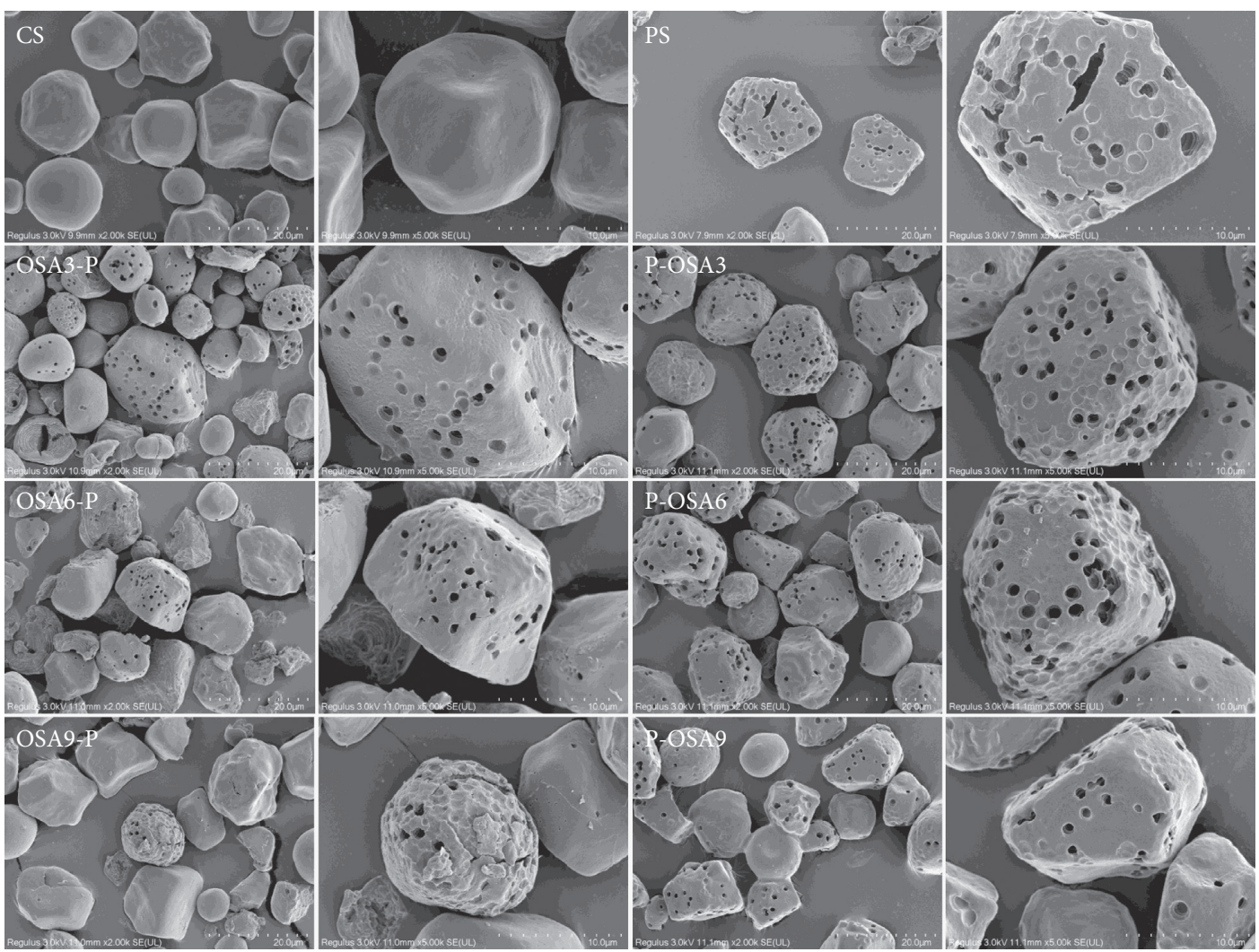

FIgURE 1: Field-emission micrographs of CS, PS, P-OSA, and OSA-P.

TABLE 2: Specific surface area and pore size of CS, PS, P-OSA, and OSA-P.

\begin{tabular}{lcr}
\hline Samples & Specific surface area $\left(\mathrm{m}^{2} / \mathrm{g}\right)$ & Pore size $(\mathrm{nm})$ \\
\hline CS & $0.516 \pm 0.018^{\mathrm{e}}$ & - \\
PS & $0.980 \pm 0.019^{\mathrm{a}}$ & $3.059 \pm 0.034^{\mathrm{b}}$ \\
P-OSA3 & $0.621 \pm 0.004^{\mathrm{cd}}$ & $3.395 \pm 0.016^{\mathrm{a}}$ \\
OSA3-P & $0.619 \pm 0.018^{\mathrm{cd}}$ & $3.400 \pm 0.033^{\mathrm{a}}$ \\
P-OSA6 & $0.651 \pm 0.009^{\mathrm{c}}$ & $3.398 \pm 0.014^{\mathrm{a}}$ \\
OSA6-P & $0.613 \pm 0.008^{\mathrm{d}}$ & $3.400 \pm 0.036^{\mathrm{a}}$ \\
P-OSA9 & $0.845 \pm 0.012^{\mathrm{b}}$ & $3.073 \pm 0.061^{\mathrm{b}}$ \\
OSA9-P & $0.606 \pm 0.006^{\mathrm{d}}$ & $3.061 \pm 0.014^{\mathrm{b}}$ \\
\hline
\end{tabular}

area value for OSA-P. This indicated that the formation of pores had an important effect on the change in the specific surface area. The presence of substituents had a significant hindering effect on enzymatic treatment, and this resistance was proportional to the DS of samples [16]. When esterification modification was performed first, followed by the hydrolysis of two enzymes, where the OS groups are located or nearby may not be subjected to enzymatic treatment. It was more difficult to produce pores, especially at high OSA levels, resulting in a smaller overall specific 
TABLE 3: The lamellar structural dimensions of CS, PS, P-OSA, and OSA-P obtained from SAXS analysis.

\begin{tabular}{lccccc}
\hline Samples & $q_{\max }$ & $D_{\text {bragg }}$ & $I$ (a.u.) & $\alpha$ & Peak area \\
\hline CS & $0.619 \pm 0.002^{\mathrm{b}}$ & $10.145 \pm 0.033^{\mathrm{c}}$ & $5.273 \pm 0.065^{\mathrm{a}}$ & $2.839 \pm 0.042^{\mathrm{bc}}$ & $1.881 \pm 0.038^{\mathrm{a}}$ \\
PS & $0.616 \pm 0.003^{\mathrm{bc}}$ & $10.195 \pm 0.050^{\mathrm{bc}}$ & $2.539 \pm 0.062^{\mathrm{e}}$ & $3.683 \pm 0.051^{\mathrm{a}}$ & $0.902 \pm 0.026^{\mathrm{d}}$ \\
P-OSA3 & $0.615 \pm 0.001^{\mathrm{bc}}$ & $10.211 \pm 0.017^{\mathrm{bc}}$ & $3.748 \pm 0.069^{\mathrm{c}}$ & $2.821 \pm 0.035^{\mathrm{bc}}$ & $1.311 \pm 0.041^{\mathrm{c}}$ \\
OSA3-P & $0.628 \pm 0.003^{\mathrm{a}}$ & $10.000 \pm 0.048^{\mathrm{d}}$ & $3.842 \pm 0.067^{\mathrm{c}}$ & $3.501 \pm 0.044^{\mathrm{a}}$ & $1.416 \pm 0.057^{\mathrm{c}}$ \\
P-OSA6 & $0.619 \pm 0.002^{\mathrm{b}}$ & $10.145 \pm 0.033^{\mathrm{c}}$ & $2.672 \pm 0.054^{\mathrm{e}}$ & $2.463 \pm 0.036^{\mathrm{cd}}$ & $1.318 \pm 0.036^{\mathrm{c}}$ \\
OSA6-P & $0.589 \pm 0.001^{\mathrm{d}}$ & $10.662 \pm 0.018^{\mathrm{a}}$ & $4.363 \pm 0.072^{\mathrm{b}}$ & $2.926 \pm 0.033^{\mathrm{b}}$ & $1.594 \pm 0.043^{\mathrm{b}}$ \\
P-OSA9 & $0.612 \pm 0.002^{\mathrm{c}}$ & $10.261 \pm 0.034^{\mathrm{b}}$ & $3.530 \pm 0.066^{\mathrm{d}}$ & $2.356 \pm 0.036^{\mathrm{d}}$ & $1.330 \pm 0.027^{\mathrm{c}}$ \\
OSA9-P & $0.616 \pm 0.002^{\mathrm{bc}}$ & $10.195 \pm 0.033^{\mathrm{bc}}$ & $5.417 \pm 0.079^{\mathrm{a}}$ & $2.922 \pm 0.031^{\mathrm{b}}$ & $1.968 \pm 0.033^{\mathrm{a}}$ \\
\hline
\end{tabular}

surface area. Moreover, the lower specific surface area resulted in weaker absorption performance of OSA-P samples than P-OSA.

3.4. Laminar Structure Analysis. The SAXS scattering profiles of starch with different treatment sequences and different OSA amounts are shown in Supplementary Figure 1. The peak position of each starch was approximately $0.6 \mathrm{~nm}^{-1}$, and the peak was generated by the alternation of crystalline and amorphous layers inside the starch granule [20]. According to the Wulff-Bragg formula $\left[D_{\text {bragg }}=2 \pi / q\right]$, the thickness of the semicrystalline lamellae of starch granules, also called the Bragg layer spacing, can be calculated [21]. In particular, after esterification and enzymatic treatment, the thickness of the starch semicrystalline lamellae $D_{\text {bragg }}$ was between 10.000 and $10.662 \mathrm{~nm}$ (Table 3).

The peak intensity $I$ at $q_{\text {max }}$ reflected the consistency of the double helix structure arrangement in the crystalline region of the starch lamellar structure, and the peak intensity decreased after enzymatic action. This was due to the fact that enzymes more easily attack the accessible and amorphous lamellae and growth rings of starch, whereas more crystalline and organized structures remain unaltered.

The fractal theory could be used to describe the selfsimilarity of the aggregate structure between geometrically ordered and geometrically disordered structures in starch granules [22]. The $\alpha$ values of each curve were calculated using Power's theorem: $I \sim q^{-\alpha}$ (shown in Table 3 ). The $\alpha$ values of P-OSA were generally smaller than those of OSA-P at the same OSA level, which implies a sparser and less ordered aggregate structure.

The area of the peak at $q_{\max }$ was calculated, with larger peak area indicating that the degree of ordering in the crystalline region of the lamellar structure was higher. The peak areas of P-OSA were generally smaller than those of OSA-P, suggesting that the enzymatic treatment disrupted the order of the crystalline region of the lamellar structure of the starch, but the degree of ordering did not easily decrease when esterified starch was subjected to enzymatic action.

3.5. Relative Crystallinity. The X-ray diffraction spectra and relative crystallinity of the samples are shown in Figure 2. A typical A-type diffraction peak was shown for all starch samples, with diffraction peaks at $15^{\circ}, 17^{\circ}, 18^{\circ}$, and $23^{\circ}$, respectively. Minor differences between the esterified modified porous starch samples and PS indicated that the esterification reaction does not change the crystalline type of starch.
The sharper diffraction peak of PS compared to CS implies that the enzymatic hydrolysis process reduces the amorphous region, which is also indicated by the increase in relative crystallinity [23]. The relative crystallinity of the samples decreased slightly after the esterification modification, but the difference between the samples with different OSA additions was not obvious, which indicated that the crystalline morphology of the starch granules changed less during the esterification modification [24-26]. Comparison of the samples obtained by reversing the order of action shows that the relative crystallinity of P-OSA was higher than that of OSA-P, which indicates a more effective enzymatic hydrolysis of P-OSA during the action. Such more efficient enzymatic hydrolysis resulted in P-OSA having a higher specific surface area than OSA-P at the same OSA addition and led to a superior absorption capacity of P-OSA.

3.6. Distribution of Octenyl Succinic Groups on Starch Granules. Confocal Raman spectroscopy can be used to assess the internal vibrations of molecules; thus, it can be used to characterize the distribution of OS groups on starch granules.

For starch granules, the characteristic peaks at $1,300-800 \mathrm{~cm}^{-1}$ are derived from the absorption peaks of native starch molecules. Supplementary Figure 2 shows that the starch modified by esterification had a characteristic peak at $1,670 \mathrm{~cm}^{-1}$ caused by the carbonyl stretching vibration in the OS groups, indicating the presence of a carbonyl group. The distribution of the OS groups on the whole starch granule can be effectively characterized by scanning the whole starch granule, layer by layer, within an $X-Y$ two-dimensional Raman spectral scan matrix under $532 \mathrm{~nm}$ laser excitation with the carbonyl group characteristic peak at $1,670 \mathrm{~cm}^{-1}$ as the target peak. Figure 3(a) shows the two-dimensional Raman spectra of OSA starch. The distribution of OS groups on the whole starch granules was not uniform, and their concentration on the surface of OSA starch granules was higher than that on the inside $[16,19]$. The distribution of OS groups in P-OSA was more uniform than that in OSA starch, and the signal of the OS groups inside the starch granules was enhanced (Figures 3(a) and $3(b)$ ), indicating that the presence of pores facilitated the reaction inside the granules during the esterification process. Figure 3(c) shows the Raman spectra of OSA-P. Overall, the distribution of OS groups in OSA-P was similar to that of OSA starch. The two-dimensional Raman spectra of the remaining samples are presented in Supplementary Figure 3. 


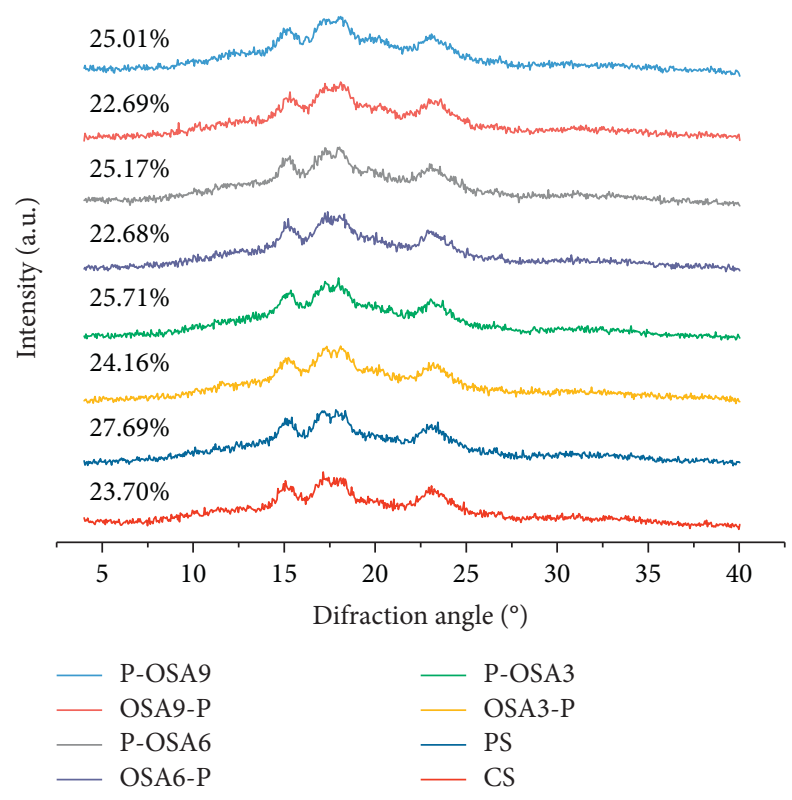

FIGURE 2: XRD patterns and relative crystallinity.
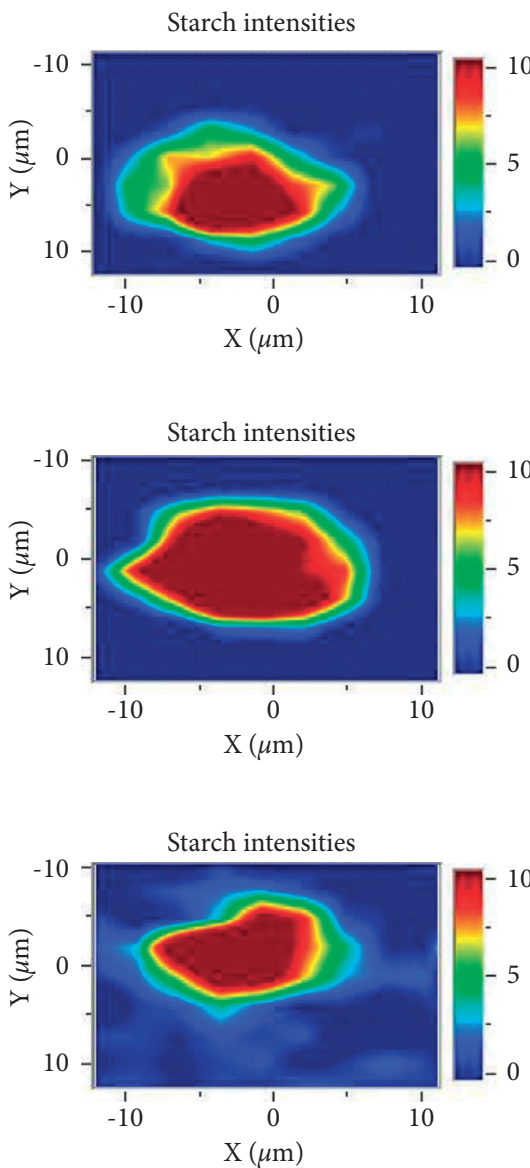

OS intensities

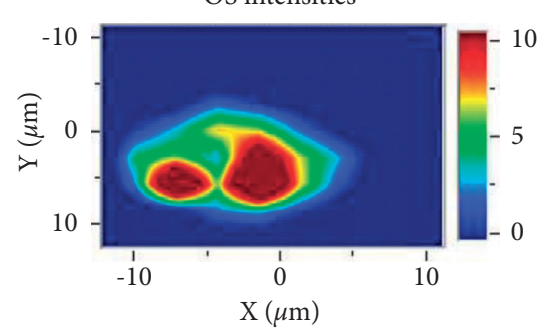

(a)

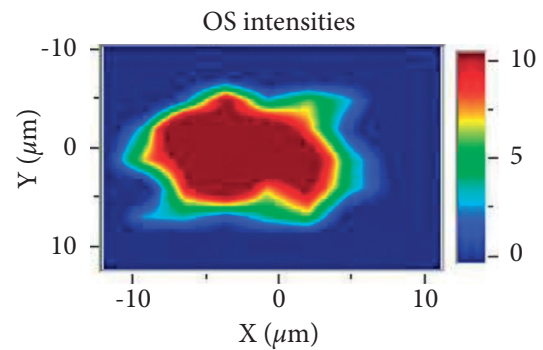

(b)

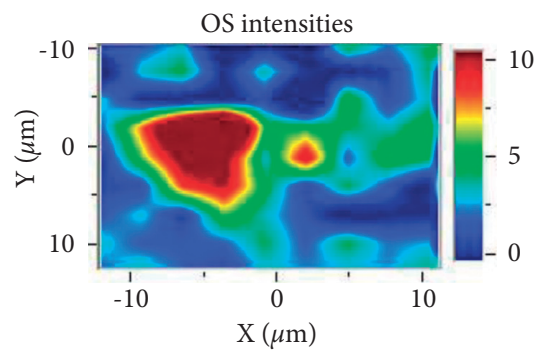

(c)
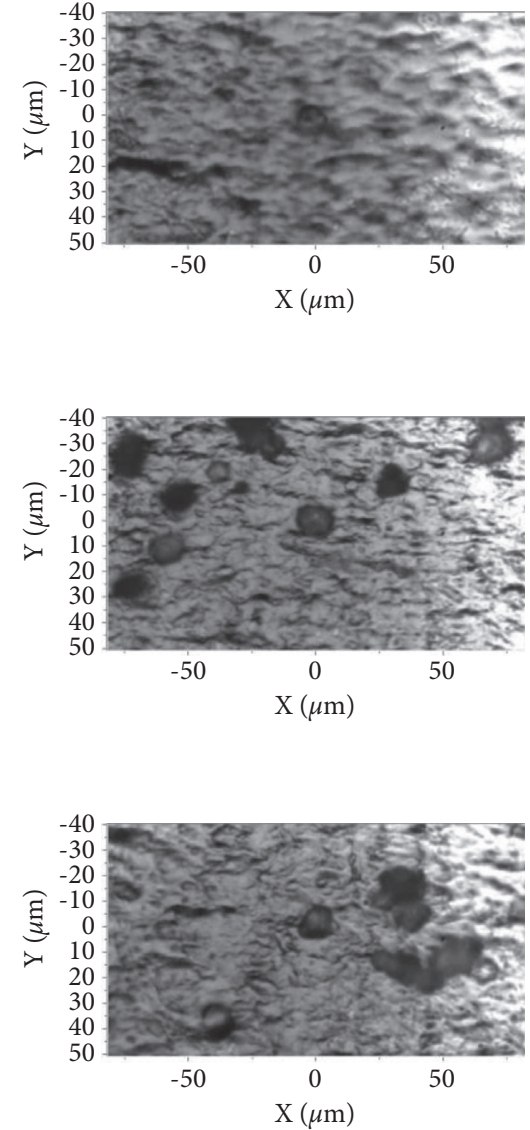

Figure 3: 2D Raman spectra of OSA9 (a), P-OSA9 (b), and OSA9-P (c). 


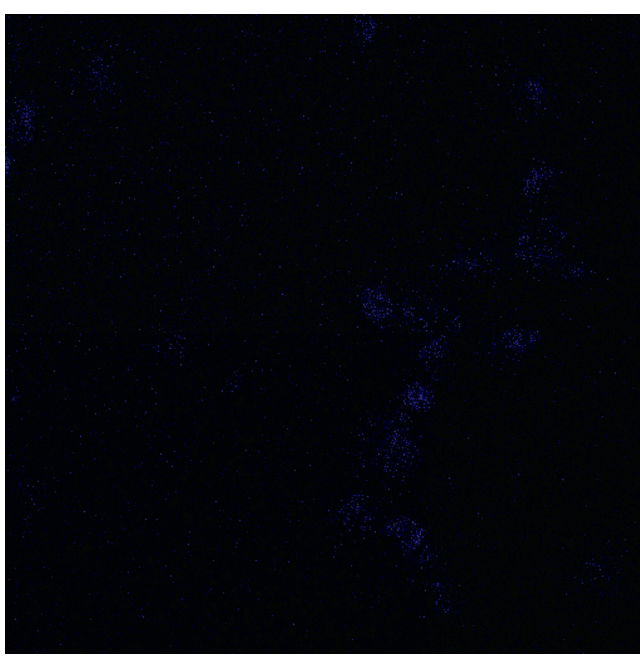

(a)

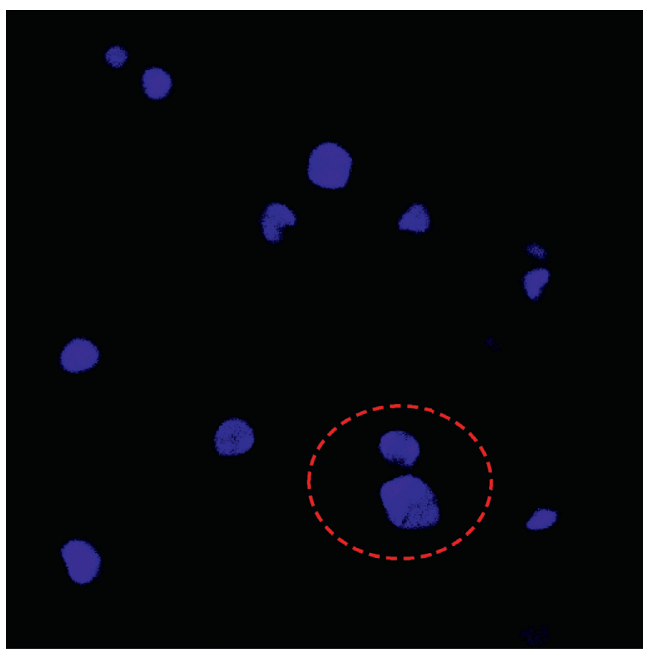

(c)

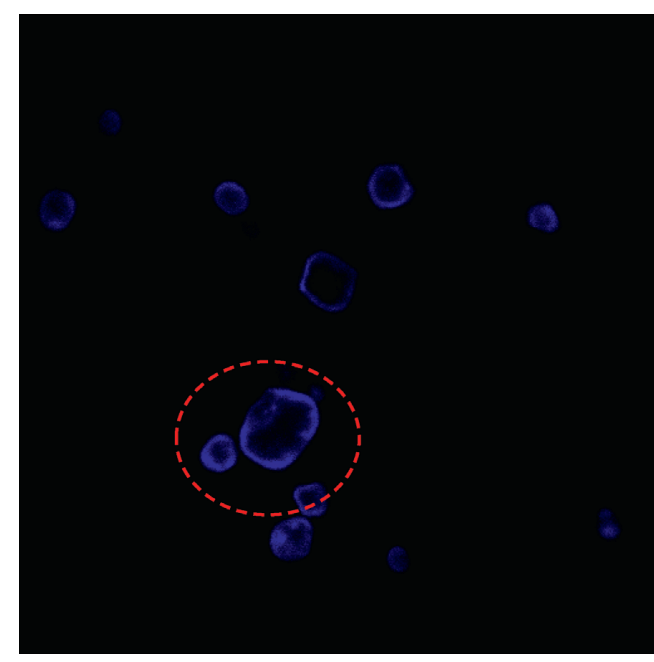

(b)

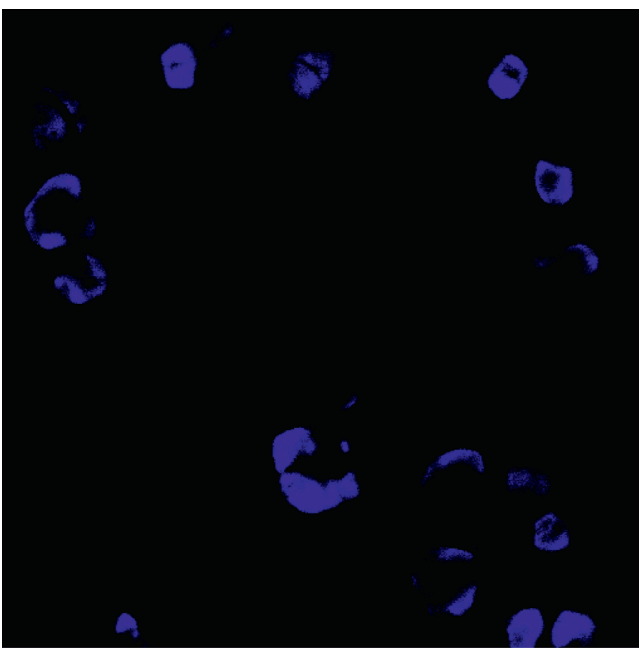

(d)

FIgURE 4: Laser confocal microscopy images of PS (a), OSA9 (b), P-OSA9 (c), and OSA9-P (d).

The laser confocal microscopy images are shown in Figure 4. Starch without OSA modification could not be stained by MB because it did not have OS groups, so the fluorescence intensity was very low. In contrast, the starch granules with OS groups were positively stained with $\mathrm{MB}$ (Figures 4(b) and 4(d)), further demonstrating that the OS groups were mainly distributed on the surface of the granules, as the fluorescence intensity inside the granules was weaker than that on the surface. The P-OSA internal part of the granules also showed fluorescent signal (Figure 4(c)), suggesting that the distribution of OS groups was affected when the porous starch was esterified, with the presence of pores being beneficial for the reaction to occur inside the granules. OS groups were not only mainly distributed on the surface of P-OSA granules, but there were also more OS groups inside the granules compared to the OSA starch.

3.7. Analysis of Menthone Loading. Under the action of enzymes, the pores on the surface of starch increased and the loading of menthone increased, and the PS loading of
TABle 4: Menthone load of CS, PS, P-OSA, and OSA-P.

\begin{tabular}{lc}
\hline Samples & Loading $(\mathrm{mg} / \mathrm{g})$ \\
\hline CS & $7.72 \pm 0.80^{\mathrm{e}}$ \\
PS & $31.83 \pm 0.41^{\mathrm{d}}$ \\
P-OSA3 & $31.80 \pm 0.88^{\mathrm{d}}$ \\
P-OSA6 & $52.76 \pm 1.09^{\mathrm{b}}$ \\
P-OSA9 & $64.34 \pm 1.23^{\mathrm{a}}$ \\
OSA3-P & $46.57 \pm 0.69^{\mathrm{c}}$ \\
OSA6-P & $47.11 \pm 1.67^{\mathrm{c}}$ \\
OSA9-P & $51.30 \pm 0.96^{\mathrm{b}}$ \\
\hline
\end{tabular}

menthone reached 4 times of CS (Table 4). The OSA modification of porous starch further increased the loading of menthone due to the increased hydrophobicity. The sequential comparison showed that except for the low OSA addition (3\%), the P-OSA had higher loading of menthone than OSA-P with the same OSAesterification addition. At high OSA addition, P-OSA showed a higher loading of menthone, indicating that P-OSA possesses the potential for menthone loading applications. 


\section{Conclusion}

Hydrophobic porous starch with different OSA amounts was prepared by the hydrolysis of amyloglucosidase and $\alpha$-amylase and OSA esterification modification, and the structures and properties of the hydrophobic porous starch obtained by different treatment sequences were compared. With the subsistence of OS groups, the hydrophobicity of starch was enhanced, which in turn improved the affinity of starch for oil. The DS of OSA-P was generally lower than that of P-OSA, which affected the absorption performance of the samples. The presence of more OS groups could hinder enzyme activity, thus leading to differences in structure and properties of samples under different treatment sequences. The relative crystallinity of the starch and SAXS data showed that for P-OSA, the relative crystallinity was higher and the aggregated structure was less ordered compared to OSA-P. The surface of P-OSA is still widely distributed with pores, and its specific surface area was larger than that of OSA-P, resulting in a better absorption capacity. However, the OSA-P did not easily form pores due to the inhibitory effect of the OS groups on the enzyme, and the absorption capacity was not ideal. In addition, the OSA reagent may react with the inside of the starch granules during the preparation process due to the presence of the pores of PS, resulting in a more uniform distribution of OS groups in the starch granules of P-OSA. The results of loading on menthone showed that P-OSA showed higher loading on menthone at high OSA additions, indicating that P-OSA possesses the potential to load menthone for application.

\section{Abbreviations}

DS: $\quad$ Degree of substitution

FESEM: Field-emission scanning electron microscopy

MB: $\quad$ Methylene blue

OS: $\quad$ Octenyl succinic group

OSA: Octenyl succinic anhydride

CS: $\quad$ Native corn starch

PS: $\quad$ Porous starch

OSAS: OSA starch

P-OSA: Starch modified by enzymatic hydrolysis followed by OSA

OSA-P: Starch modified by OSA followed by enzymatic hydrolysis. The numbers following OSA (3, 6, and 9) represent the amount of OSA added as a percentage of the dry starch weight $(\mathrm{w} / \mathrm{w})$ during preparation.

\section{Data Availability}

The data used to support the findings of this study are included within the article.

\section{Conflicts of Interest}

The authors declare that they have no conflicts of interest.

\section{Acknowledgments}

This research was supported by the National Natural Science Foundation of China (nos. 31571794 and 31560437), National First-Class Discipline Program of Food Science and Technology (JUFSTR20180204), Science and Technology Major Project of Guangxi (no. guikeAA17202029), and Postgraduate Research \& Practice Innovation Program of Jiangsu Province (no. KYCX21_2046).

\section{Supplementary Materials}

Supplementary Figure 1: the $I \sim q$ curves of CS, PS, P-OSA, and OSA-P obtained from SAXS analysis. Supplementary Figure 2: Raman spectra of OSAS. Supplementary Figure 3: 2D Raman spectra of OSA3 (a), P-OSA3 (b), OSA3-P (c), OSA6 (d), P-OSA6 (e), and OSA6-P (f). Supplementary Figure 4: the morphology of samples after oil absorption. Supplementary Figure 5: the morphology of samples after MB absorption. Supplementary Figure 6: field-emission micrographs of samples after MB absorption. Supplementary Figure 7: absorption amount in pre-experiment. Supplementary Figure 8: field-emission micrographs of CS and PS in pre-experiment. Supplementary Table 1: absorption properties and DS of samples with the same degree of esterification and different enzyme additions and order of action. (Supplementary Materials)

\section{References}

[1] X. Ma, X. Liu, D. P. Anderson, and P. R. Chang, "Modification of porous starch for the adsorption of heavy metal ions from aqueous solution," Food Chemistry, vol. 181, pp. 133-139, 2015.

[2] Y. Xie, M.-N. Li, H.-Q. Chen, and B. Zhang, "Effects of the combination of repeated heat-moisture treatment and compound enzymes hydrolysis on the structural and physicochemical properties of porous wheat starch," Food Chemistry, vol. 274, pp. 351-359, 2019.

[3] J. Liu, X. Wang, H. Yong, J. Kan, and C. Jin, "Recent advances in flavonoid-grafted polysaccharides: synthesis, structural characterization, bioactivities and potential applications," International Journal of Biological Macromolecules, vol. 116, pp. 1011-1025, 2018.

[4] J. Chen, Y. Wang, J. Liu, and X. Xu, "Preparation, characterization, physicochemical property and potential application of porous starch: a review," International Journal of Biological Macromolecules, vol. 148, pp. 1169-1181, 2020.

[5] Q.-Q. Lv, G.-Y. Li, Q.-T. Xie et al., "Evaluation studies on the combined effect of hydrothermal treatment and octenyl succinylation on the physic-chemical, structural and digestibility characteristics of sweet potato starch," Food Chemistry, vol. 256, pp. 413-418, 2018.

[6] P. R. Chang, D. Qian, D. P. Anderson, and X. Ma, "Preparation and properties of the succinic ester of porous starch," Carbohydrate Polymers, vol. 88, no. 2, pp. 604-608, 2012.

[7] Y. Bai, R. C. Kaufman, J. D. Wilson, and Y.-C. Shi, "Position of modifying groups on starch chains of octenylsuccinic anhydride-modified waxy maize starch," Food Chemistry, vol. 153, pp. 193-199, 2014.

[8] Y. Bai and Y.-C. Shi, "Structure and preparation of octenyl succinic esters of granular starch, microporous starch and 
soluble maltodextrin," Carbohydrate Polymers, vol. 83, no. 2, pp. 520-527, 2011.

[9] B. Zhang, D. Cui, M. Liu, H. Gong, Y. Huang, and F. Han, "Corn porous starch: preparation, characterization and adsorption property," International Journal of Biological Macromolecules, vol. 50, no. 1, pp. 250-256, 2012.

[10] X.-y. Song, Q.-h. Chen, H. Ruan, G.-q. He, and Q. Xu, "Synthesis and paste properties of octenyl succinic anhydride modified early Indica rice starch," Journal of Zhejiang University-Science $B$, vol. 7, no. 10, pp. 800-805, 2006.

[11] Y. Xie, B. Zhang, M.-N. Li, and H.-Q. Chen, "Effects of crosslinking with sodium trimetaphosphate on structural and adsorptive properties of porous wheat starches," Food Chemistry, vol. 289, pp. 187-194, 2019.

[12] Y. Zheng, Y. Ou, C. Zhang et al., "The impact of various exogenous type starch on the structural properties and dispersion stability of autoclaved lotus seed starch," International Journal of Biological Macromolecules, vol. 175, pp. 49-57, 2021.

[13] R. Bardestani, G. S. Patience, and S. Kaliaguine, "Experimental methods in chemical engineering: specific surface area and pore size distribution measurements-BET, BJH, and DFT," Canadian Journal of Chemical Engineering, vol. 97, no. 11, pp. 2781-2791, 2019.

[14] Z. Yang, X. Xu, Y. Hemar, G. Mo, L. de Campo, and E. P. Gilbert, "Effect of porous waxy rice starch addition on acid milk gels: structural and physicochemical functionality," Food Hydrocolloids, vol. 109, Article ID 106092, 2020.

[15] X. Chen, R. Liang, F. Zhong et al., "Effect of high concentrated sucrose on the stability of OSA-starch-based beta-carotene microcapsules," Food Hydrocolloids, vol. 113, Article ID 105472, 2021.

[16] B. Zhang, Q. Huang, F.-x. Luo, X. Fu, H. Jiang, and J.-1. Jane, "Effects of octenylsuccinylation on the structure and properties of high-amylose maize starch," Carbohydrate Polymers, vol. 84, no. 4, pp. 1276-1281, 2011.

[17] W. Gao, J. Sui, P. Liu, B. Cui, and A. M. Abd El-Aty, "Synthetic mechanism of octenyl succinic anhydride modified corn starch based on shells separation pretreatment," International Journal of Biological Macromolecules, vol. 172, pp. 483-489, 2021.

[18] Y. Benavent-Gil and C. M. Rosell, "Morphological and physicochemical characterization of porous starches obtained from different botanical sources and amylolytic enzymes," International Journal of Biological Macromolecules, vol. 103, pp. 587-595, 2017.

[19] Q. Huang, X. Fu, X.-w. He, F.-x. Luo, S.-j. Yu, and L. Li, “The effect of enzymatic pretreatments on subsequent octenyl succinic anhydride modifications of cornstarch," Food $\mathrm{Hy}$ drocolloids, vol. 24, no. 1, pp. 60-65, 2010.

[20] J. Blazek and E. P. Gilbert, "Application of small-angle X-ray and neutron scattering techniques to the characterisation of starch structure: a review," Carbohydrate Polymers, vol. 85, no. 2, pp. 281-293, 2011.

[21] L. Zhang, X. Li, S. Janaswamy, L. Chen, and C. Chi, "Further insights into the evolution of starch assembly during retrogradation using SAXS," International Journal of Biological Macromolecules, vol. 154, pp. 521-527, 2020.

[22] T. Suzuki, A. Chiba, and T. Yarno, "Interpretation of small angle x-ray scattering from starch on the basis of fractals," Carbohydrate Polymers, vol. 34, no. 4, pp. 357-363, 1997.

[23] L. Guo, J. Li, Y. Gui et al., "Porous starches modified with double enzymes: structure and adsorption properties,"
International Journal of Biological Macromolecules, vol. 164, pp. 1758-1765, 2020.

[24] B. Zhang, J.-Q. Mei, B. Chen, and H.-Q. Chen, "Digestibility, physicochemical and structural properties of octenyl succinic anhydride-modified cassava starches with different degree of substitution," Food Chemistry, vol. 229, pp. 136-141, 2017.

[25] H. Hu, W. Liu, J. Shi et al., "Structure and functional properties of octenyl succinic anhydride modified starch prepared by a non-conventional technology," Starch-Stärke, vol. 68, no. 1-2, pp. 151-159, 2015.

[26] Z. Yi, L. Hu, D. Ning, L. Ping, and H. Zhang, "Physicochemical and structural characteristics of the octenyl succinic ester of ginkgo starch," International Journal of Biological Macromolecules, vol. 94, pp. 566-570, 2017. 